\section{Diagnosis of carotid artery stenosis by noninvasive imaging}

Safer, noninvasive imaging techniques are replacing intra-arterial angiography for the assessment of carotid artery stenosis. To assess the accuracy of the various noninvasive methods, Wardlaw et al. conducted a metaanalysis of trials comparing these imaging techniques with intra-arterial angiography.

Of 672 studies published from January 1980 to April 2004, 41 met the inclusion criteria for the study; papers were excluded because of subsequent technological advances, incomplete data, or poor methodology. For the detection of $70-99 \%$ stenosis, contrastenhanced magnetic resonance angiography (MRA) had the highest sensitivity (94\%) of the techniques examined, followed by Doppler ultrasound (89\%), non-contrast-enhanced MRA (88\%) and CT angiography (77\%). CT angiography, however, was found to have the highest specificity (95\%), followed by contrastenhanced MRA (93\%), Doppler ultrasound (84\%) and MRA (84\%). For 50-69\% stenosis, reliable comparisons could not be made because of insufficient data, but the accuracy of all methods was considerably reduced.

The authors state that noninvasive imaging techniques are accurate for the diagnosis of $70-99 \%$ stenosis, but additional carefully designed studies are required to determine the accuracy of these techniques in moderate stenosis. Pre-screening of patients with Doppler ultrasound introduced verification bias to the studies. Most trials involved patients with an average age in the 60s, whereas trials in older patients would be more relevant to stroke prevention. The authors conclude that prospective, properly blinded studies in older patients are necessary to determine the best procedure for the diagnosis of carotid artery stenosis.

Original article Wardlaw JM et al. (2006) Non-invasive imaging compared with intra-arterial angiography in the diagnosis of symptomatic carotid stenosis: a meta-analysis. Lancet 367: 1503-1512

\section{Tumor vascularity predicts prognosis in adult, but not pediatric, astrocytomas}

Astrocytomas are the most common form of brain tumor in both adults and children, and are associated with a poor prognosis. A Turkish group has investigated whether tumor vascularity can predict prognosis in adults and children with this tumor type.

Birlik and colleagues enrolled 45 adults (aged 19-82 years) and 25 children (aged $1-16$ years) with astrocytomas. They determined the vascularity of the tumors according to microvessel density, vascular grading and Chalkley counting. During a median follow-up of 12-24 months, 22 adults and 4 children died from disease progression. In univariate analysis, all three tumor vascularity parameters were significantly associated with postoperative survival in adults $(P \leq 0.005$ for all). Higher-grade tumor histology in adults and older age in the overall group also correlated with reduced survival. In multivariate analysis, microvessel density was a significant independent prognostic indicator $(P=0.001)$; adults with microvessel density $\geq 70$ per $200 \times$ field had significantly shorter survival than those with lower microvessel densities $(P<0.001)$. By contrast, tumor histology and vascular parameters were not significantly associated with survival in children. The authors suggest that the observed differences between adults and children probably arise from biological variation in patients of different ages, relating to the ability of different tumors to induce angiogenesis.

The authors conclude that quantification of tumor vascularity may be useful in planning and monitoring antiangiogenic therapies for adult astrocytomas, but might not be of benefit in pediatric astrocytomas. This hypothesis needs confirming in further studies with longer follow-up in pediatric patients with high-grade astrocytomas.

Original article Birlik B et al. (2006) Tumour vascularity is of prognostic significance in adult, but not paediatric astrocytomas. Neuropathol Appl Neurobiol

[doi: 10.1111/j.1365-2990.2006.00763.x] 